\title{
Effect of Substrate Choice and Tissue Type on Tissue Preparation for Spectral Histopathology by Raman Microspectroscopy
}

\author{
Hugh Byrne \\ Technological University Dublin, hugh.byrne@tudublin.ie \\ Leanne M. Fullwood \\ University of Central Lancashire \\ Dave Griffiths \\ University of Central Lancashire
}

See next page for additional authors

Follow this and additional works at: https://arrow.tudublin.ie/biophonart

Part of the Physics Commons

\section{Recommended Citation}

Byrne, H. J. et al (2014) Effect of Substrate Choice and Tissue Type on Tissue Preparation for Spectral Histopathology by Raman Microspectroscopy", Analyst, 139, pp.446 - 454. DOI: 10.1039/C3AN01832F

This Article is brought to you for free and open access by the DIT Biophotonics and Imaging at ARROW@TU Dublin. It has been accepted for inclusion in Articles by an authorized administrator of ARROW@TU Dublin. For more information, please contact arrow.admin@tudublin.ie, aisling.coyne@tudublin.ie,gerard.connolly@tudublin.ie. Funder: HEA

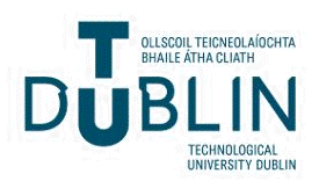




\section{Authors}

Hugh Byrne, Leanne M. Fullwood, Dave Griffiths, Katherine Ashton, Timothy Dawson, Robert W. Lea, Charles Davis, Franck Bonnier, and Matthew J. Baker 


\title{
Effect of Substrate Choice and Tissue Type on Tissue Preparation for Spectral Histopathology by Raman Microspectroscopy
}

\author{
Leanne M. Fullwood ${ }^{1}$, Dave Griffiths ${ }^{2}$, Katherine Ashton ${ }^{3}$, Timothy Dawson ${ }^{3}$, Robert W. \\ Lea $^{2}$, Charles Davis ${ }^{3}$, Franck Bonnier ${ }^{4}$, Hugh J. Byrne ${ }^{4}$, and Matthew J. Baker ${ }^{1 *}$ \\ ${ }^{1}$ Centre for Materials Science, Division of Chemistry, University of Central Lancashire, Preston, PR1 2HE, UK \\ ${ }^{2}$ School of Pharmacy and Biomedical Sciences, University of Central Lancashire, Preston, PR1 2HE, UK \\ ${ }^{3}$ Lancashire Teaching Hospital NHS Trust Royal Preston Hospital, Sharoe Green Lane, Preston PR2 9HT, UK \\ ${ }^{4}$ FOCAS Research Institute, Dublin Institute of Technology, Kevin Street, Dublin 8, Ireland \\ *Corresponding Author: mjbaker@uclan.ac.uk
}

\section{Abstract}

Raman spectroscopy is a non-destructive, non-invasive, rapid and economical technique which has the potential to be an excellent method for the diagnosis of cancer and understanding disease progression through retrospective studies of archived tissue samples. Historically, biobanks are generally comprised of formalin fixed paraffin preserved tissue and as a result these specimens are often used in spectroscopic research. Tissue in this state has to be dewaxed prior to Raman analysis to reduce paraffin contributions in the spectra. However, although the procedures are derived from histopathological clinical practice, the efficacy of the dewaxing procedures that are currently employed is questionable. Ineffective removal of paraffin results in corruption of the spectra and previous experiments have shown that the efficacy can depend on the dewaxing medium and processing time. The aim of this study was to investigate the influence of commonly used spectroscopic substrates $\left(\mathrm{CaF}_{2}\right.$, Spectrosil quartz and low-E slides) and the influence of different histological tissue types (normal, cancerous and metastatic) on tissue preparation and to assess their use for spectral 
histopathology. Results show that $\mathrm{CaF}_{2}$ followed by Spectrosil contribute the least to the spectral background. However, both substrates retain paraffin after dewaxing. Low-E substrates, which exhibit the most intense spectral background, do not retain wax and resulting spectra are not affected by paraffin peaks. We also show a disparity in paraffin retention depending upon the histological identity of the tissue with abnormal tissue retaining more paraffin than normal.

Keywords Cancer, Diagnosis, Raman, Substrate, Histopathology, Vibrational Spectroscopy 


\section{Introduction}

The primary requirement for successful treatment of any disease is early detection. Cancer incidence rates have continued to rise ${ }^{1}$, although survival rates for cancer are increased with early diagnosis. Current methods used in cancer diagnosis focus on changes in architecture of tissue, cells or internal constituents and the identification of protein expression. Research performed into identification of protein expression in blood has produced single biomarkers for specific cancers that have become misleading. There have been many studies of different pathologies but the issues are well demonstrated by the example of prostate cancer. The Prostate Specific Antigen (PSA) test is used to indicate prostate cancer $(\mathrm{CaP})$. However, approximately $40 \%$ of organ confined $\mathrm{CaP}$ show no elevation of PSA ${ }^{2}$ and many other benign conditions can show increased PSA serum, thus producing false positives in screening. Hoffman et al. suggest that $\mathrm{CaP}$ screening would benefit from improved biomarkers, which more readily identify clinically important cancers ${ }^{3}$. As cancer is a heterogeneous disease, a set of markers or a whole sample profile will provide significantly more diagnostic information than any one marker ${ }^{4}$. After initial indication of cancer, diagnosis is confirmed by biopsy and assessment of tissue architecture. The biopsy is assessed based upon a subjective measurement. For $\mathrm{CaP}$ this is called the Gleason grading system and in a study of 390 patients identical grades were assigned to only $29.2 \%$ of the tumours by different histopathologists ${ }^{5}$. Pathological discrepancies are reported to occur in up to $43 \%$ of specimens assessed via histopathology ${ }^{6}$. The accurate and rapid diagnosis of disease allows early intervention of appropriate treatment, thus increasing life expectancy and reducing healthcare costs ${ }^{7}$. Therefore, there is a requirement for nonsubjective techniques that can rapidly and accurately identify disease. 
Spectroscopic techniques, such as Raman and Infrared (IR), are excellent methods for the analysis of a wide range of materials. They are non-destructive, rapid, cost-effective and simple to operate. The inelastic (Raman) scattered light from molecules under irradiation is wavelength-shifted with respect to the incident light by molecular vibrations. The Raman spectrum is complementary to that of IR which represents the absorption of incident light at the resonant frequency of the bond or group, exciting vibrational modes. Different biomolecules exhibit responses to different wavelengths of light; the resultant spectrum can be thought of as a 'fingerprint' of the sample. Spectroscopic analysis allows the objective classification of biological material on a molecular level ${ }^{8}$. Previous studies have demonstrated the use of spectroscopy (imaging and point spectra) to analyse gastrointestinal (GI) $\operatorname{tract}^{9,10}$, lung ${ }^{11,12}$, cervix ${ }^{13,14}$, brain ${ }^{15,16}$,breast ${ }^{17,18}$, prostate ${ }^{19-21}$ and lymph node cancers ${ }^{22,23}$. Initial work has focused on differentiating between normal tissue and advanced cancers. Technological development is enabling diagnosis at progressively earlier stages and potential to do so in vivo ${ }^{24}$. The aim of these studies is to develop spectral histopathology based upon objective chemical information.

In order to develop spectral histopathology, a database of tissue spectra is required to enable spectral disease identification. The ideal situation would be to build this database using fresh or frozen tissue sections. However, historically, biobanks have been built using Formalin Fixed Paraffin Preserved Tissue (FFPP) sections, which are fixed using formalin and impregnated with paraffin wax to preserve the tissue for future analysis and provide support to aid microtomy for sectioning the tissue block to enable microscopic examination. Prior to histological and/or spectral analysis, the tissue sections generally undergo a 
dewaxing process, to allow histological staining of the tissue for pathological examination and to reduce paraffin contributions in vibrational spectra, consequently, returning the tissue to as much of an in vivo state as possible ${ }^{25}$.

Ó Faoláin et al. investigated the efficacy of dewaxing procedures on formalin fixed, paraffin preserved (FFPP) cervical tissue on glass slides ${ }^{25}$. They reported that dewaxing procedures employing, the commonly used xylene and HistoClear solvents do not completely remove all of the paraffin wax. Although, they found hexane to be a much more efficient dewaxing agent, it requires 18 hours of tissue submersion and is not clinically used for dewaxing procedures ${ }^{25}$. An important advantage in using spectroscopy for diagnosis is the short diagnostic window; therefore, a dewaxing solvent that requires a long processing time period is not an attractive option for this field of study. Ó Faoláin et al. indicate that Raman spectroscopy is much more sensitive to assess the incomplete removal of wax than FTIR. This was concluded following analysis of Raman and FTIR spectra obtained from identically deparaffinised tissue sections in which spectral peaks characteristic of paraffin wax were more clearly resolved in Raman spectra (strong sharp bands), compared with FTIR spectra ${ }^{26}$.

The contribution of paraffin peaks in vibrational spectra is apparent in both infrared and Raman spectroscopy. However, these peaks appear more prominently in the spectra of the latter technique; thus, Raman spectroscopy was used in this study to analyse the efficacies of current dewaxing techniques.

Substrates are needed in spectral histopathology to support the samples during analysis. Samples are often relatively thin allowing incident light the opportunity of interacting with the substrate below. This interaction has an effect on the spectral 
background; thus, the correct choice of substrate for spectroscopic analysis is essential for reducing this background and optimising the quality of the spectral data ${ }^{27}$. Commercial glass microscope slides exhibit a very large fluorescent background under excitation in the near infrared, and are thus not suitable. Therefore, common spectroscopy substrates currently used include quartz, $\mathrm{CaF}_{2}$ and low-E microscope slides, the latter being relatively low cost. ${ }^{28}$

Recently, the choice of substrate has been a hot topic within the spectral histopathology field, as spectral artefacts have been identified in FTIR transflection analysis using highly reflective low-E slides ${ }^{29,30}$. However, when analysing tissue, a $2^{\text {nd }}$ derivative spectrum minimises these contributions allowing for spectral histopathological diagnosis ${ }^{31}$. Both quartz and $\mathrm{CaF}_{2}$ are transparent over a large range of the mid-IR and are therefore suitable for transmission measurements, although $\mathrm{CaF}_{2}$ has been shown to be better for Raman measurements, and so is an obvious choice for use of both complementary techniques. The choice of substrate is also important if spectral diagnoses are going to be effective in the clinical setting, substrates that can easily fit into current methodologies (due to size, solubility etc.) are advantageous and also cost needs to be minimised to ensure efficient health services.

Vibrational spectroscopy has the potential to revolutionise the clinical environment, allowing for increased efficiency within the diagnostic regime with corresponding decreases in mortality, morbidity and economic impact upon the health services ${ }^{32}$. In order to achieve this potential, research needs to be performed to understand the impact of sample preparation upon the vibrational spectrum. We present, for the first time, a study of the effect and efficiency of the dewaxing process, using a clinical standard HistoClear procedure, by 
substrate and tissue type utilising Raman spectroscopy in order to develop the basis of standard approaches for spectral histopathology.

\section{Experimental}

\section{Study participants}

Tissue sections cut, by microtomy, at both $4 \mu \mathrm{m}$ onto glass microscope slides and 10 $\mu \mathrm{m}$ onto spectroscopic substrates: low-E MirrIR slides (Kevley Technologies, Chesterland, $\mathrm{OH}, 44026$, USA), Spectrosil quartz (Starna Scientific) and calcium fluoride $\left(\mathrm{CaF}_{2}\right)$ (Crystran), were obtained from formalin fixed paraffin preserved (FFPP) tissue blocks from the Brain Tumour North West (BTNW) bio-bank under ethical approval (BTNW/WRTB 13_01). The substrates had not been used for prior investigations and were fresh for this study. The measurements were taken from multiple slides and multiple places on each slide so batch to batch variability should not affect the trends in the data shown. The tissue sections used from each sample were cut consecutively with one section used for Raman analysis and one for haematoxylin and eosin staining. Patient data consisted of histological information, patient gender and date of birth, origin of metastasis/histological subtype. A total of 48 tissue specimens were obtained from 41 different patients. Tissue consisted of normal brain samples $(\mathrm{n}=7)$, glioblastoma multiforme (GBM) brain samples WHO (World Health Organisation) grade IV $(n=5)$ and metastatic brain samples $(n=29)$. Table 1 displays further information about the tissue specimens. 


\section{Dewaxing and Haematoxylin and Eosin staining of tissue}

Tissue sections on the microscope slides needed to undergo staining with haematoxylin and eosin (H\&E) for parallel histological examination. The sections were dewaxed before staining, carried out by: $2 \times 5$ minute baths of HistoClear followed by $2 \times 5$ minute baths of ethanol. The tissue sections were washed in distilled water for 5 minutes after de-waxing, prior to H\&E staining. Sections were bathed in haematoxylin for 5 minutes and then washed in warm tap water to allow the nuclei to turn blue. The sections were then covered in eosin for 4 minutes and rinsed with distilled water. Finally, the tissue sections were dehydrated in $2 \times 5$ minute baths of ethanol and cleared in $2 \times 5$ minute baths of histoclear, and then protected and preserved through the application of HistoMount and a coverslip. The sections were then microscopically examined in order to identify the metastatic sites present in the tissue.

Figure 1 shows the microscopic images of the $\mathrm{H} \& \mathrm{E}$ stained tissue samples at x 100 magnification. It can be observed that normal brain, metastatic and GBM brain tissue architecturally differ from one another and between the metastatic types.

\section{Tissue section preparation for Raman spectroscopic analysis}

The tissue sections on Raman substrates needed to be de-waxed prior to Raman analysis in order to reduce the paraffin peak contributions in the spectra. The de-waxing procedure consisted of $3 \times 5$ minute baths of HistoClear followed by $3 \times 5$ minute baths of 
ethanol. The sections were left to air dry for 30 minutes, placed in a Petri dish and stored in a desiccator until spectroscopic analysis.

\section{Raman spectroscopy instrumentation and analysis}

Spectroscopic measurements were carried out using a Horiba Jobin-Yvon LabRAM HR800 spectrometer. An air cooled CLDS point mode diode $785 \mathrm{~nm}$ laser with a single edge filter (cut off to $100 \mathrm{~cm}^{-1}$ ) and an output power of $300 \mathrm{~mW}$ was used to acquire spectra; which was used with a grating of $300 \mathrm{gr} / \mathrm{mm}$ and blazed at $1000 \mathrm{~nm}$. Point spectra were acquired using a x 100 (Olympus MPlanN, NA = 0.9, spotsize $\sim 1 \mu \mathrm{m}$ ) objective and immersion point spectra were taken using a x 60 (Olympus LUMPlanFLN, NA $=1.0$, spotsize $\sim 1.5 \mu \mathrm{m}$ ). Immersion spectroscopy was used for measurement of the tissue samples for spectral histopathology. Immersion Raman utilises an immersion lens which is in direct contact with a liquid that the sample under analysis is submerged in, such as deionised water used in this study. Bonnier et al. describe the use of immersion Raman spectroscopy and demonstrate its successful use for improving spectral quality ${ }^{34,35}$.

The confocal hole was set at $100 \mu \mathrm{m}$ for $785 \mathrm{~nm}$ spectral collections. The detector used was an Andor charged coupled device (CCD). A video camera within the Raman system was used to take images of the specimens. The instrumentation was calibrated before operation to silicon at the spectral line of $520.8 \mathrm{~cm}^{-1}$. Spectra were acquired using the $785 \mathrm{~nm}$ laser at $100 \%$ exposure for $30 \mathrm{~s}$ and accumulated twice. From each tissue section 20 spectra were acquired from different regions, depending upon the size of the tissue slice. 
For all tissue samples, sample analysis by spectral acquisition was carried out as it would be in the clinic. i.e all and any normal tissue was analysed, and no particular area was targeted (eg white/grey matter). Similarly, for tumoral sections, all cancerous areas were analysed irrespective of the origin of the structure, again mimicking clinical practice.

\section{Data pre-processing}

Pre-processing was carried out on the raw data using LabSpec 6 spectroscopy software suite (HORIBA Scientific, Japan) and MATLAB version 7.11.0 (R2010b) (The MathWorks, Inc., USA) using in-house written software. Pre-processing methods were kept to a minimum to enable better reproducibility; background subtracted was performed through the application of a fifth order polynomial and smoothed using 7 point smoothing (Labspec 6) and vector normalised (Matlab) using in-house written code.

\section{Atomic force microscope (AFM)}

The atomic force microscope (AFM) model that was used to image the samples for surface morphology for this study is the MFP-3D-BIO (Asylum Research, USA), with Olympus silicon AC160 cantilevers. The tips were $160 \mathrm{~nm}$ long with resonant frequencies typical of $320 \mathrm{kHz}$. A/C mode was employed for operation to reduce tip/sample.

\section{Results and Discussion}

\section{Dewaxing efficiency of spectroscopic substrates}


Raman spectra of the three substrates: low-E, Spectrosil and $\mathrm{CaF}_{2}$, were acquired under identical conditions to generate spectral 'fingerprints' of the substrates, shown in Figure 2, and thus ascertain the impact of their contribution to the spectral background.

It can be observed that low-E has the highest intensity baseline and $\mathrm{CaF}_{2}$ has the lowest intensity baseline of the three substrates; indicating that spectra taken from samples on $\mathrm{CaF}_{2}$ are less affected by substrate background than either low-E or Spectrosil and should display background peaks of lower intensities.

The spectra of dewaxed tissue samples on the three different substrates were compared to further investigate the level of influence substrate type has on the outcome of spectra. Figure 3 displays average spectra of dewaxed tissue on the three substrates and a paraffin spectrum for comparison

A paraffin spectrum displays significant peaks at $888 \mathrm{~cm}^{-1}, 1061 \mathrm{~cm}^{-1}, 1131 \mathrm{~cm}^{-1}$, $1171 \mathrm{~cm}^{-1}, 1294 \mathrm{~cm}^{-1}, 1417 \mathrm{~cm}^{-1}, 1440 \mathrm{~cm}^{-1}, 1462 \mathrm{~cm}^{-1}$ (Figure 3), which can be assigned to C-C stretching and $\mathrm{CH}_{2}$ and $\mathrm{CH}_{3}$ deformations within the molecule ${ }^{25}$. It can be seen from Figure 3 that the spectra from the dewaxed tissue samples on Spectrosil and $\mathrm{CaF}_{2}$ exhibit residual paraffin peaks. However, dewaxed tissue spectra on low-E do not display visible paraffin peaks.

In order to further investigate any substrate effect on the dewaxing process, paraffin wax was analysed on the substrates without the presence of tissue specimens. Paraffin wax was cut at $10 \mu \mathrm{m}$ sections and mounted onto the three different substrates. The substrates 
were then dewaxed by the same method as the FFPP tissue samples and analysed; Figure 4 displays the resulting spectra. Residual wax, still present on both the Spectrosil and $\mathrm{CaF}_{2}$ substrate, could be seen visually both macroscopically and microscopically, shown in Figure 5, and its presence was confirmed by paraffin peak contributions in their spectra. The low-E substrate did not retain any visual paraffin wax and no paraffin peaks were present in the 10 spectra taken from the substrate. This shows, for the first time, a substrate effect that occurs during the dewaxing process inferring that as a result of surface chemistry or morphology, low-E slides facilitate the removal of wax more readily than $\mathrm{CaF}_{2}$ and Spectrosil. From Raman analysis and visual inspection, it is clear that, using the same dewaxing protocol, the low-E substrate is fully dewaxed, yet the $\mathrm{CaF}_{2}$ and Spectrosil substrates retain paraffin wax.

Atomic force microscopy (AFM) was utilised to assess the three substrates' surface roughness. The AFM images of the three substrates are displayed in Figure 6, showing the surface morphology at a nanoscopic level. The low-E substrate was observed to have a far smoother surface than either $\mathrm{CaF}_{2}$ or Spectrosil with an average surface roughness of $1.10 \mathrm{~nm}$ compared to $4.76 \mathrm{~nm}$ and $3.88 \mathrm{~nm}$ respectively. Low-E slides are coated with metal oxide ${ }^{36}$; this could explain why their surface is far smoother than the other substrates which are only polished.

The clinical application of spectral histopathology is the ultimate aim of this field. As such, the adherence to current clinical process will be advantageous. The use of Low-E slides holds a number of advantages for this, such as low cost, their microscope slide size and robustness when compared to other substrates. Although substrate spectral background is 
higher, all tissue spectral peaks that are detected when using other substrates are present in the spectrum.

\section{Spectral Histopathology: Dewaxing Efficiency by Tissue Types}

Although low-E substrates clearly perform better in terms of wax removal from the tissue sections using routine clinical protocols, $\mathrm{CaF}_{2}$ is commonly used in Raman spectroscopic studies due to its low contribution to the spectral background (Figure 2). It is of interest to investigate whether the tissue type mounted on these substrates also contributes to the residual signals. The vector normalised mean Raman spectra of dewaxed sections of normal (157 spectra from 7 patients), metastatic (668 spectra from 29 patients) and GBM (127 spectra from 5 patients) brain tissue on $\mathrm{CaF}_{2}$ substrates are displayed in Figure 7. It can be observed from the averaged spectrum of normal tissue that the paraffin peaks (asterisked peaks) are not fully removed by the dewaxing process, but notably the characteristic features are of a much lower intensity than those in the spectra of metastatic and GBM tissue Figure 8 shows the average intensity and standard deviation of the intense paraffin peaks at 1061, 1131 and $1294 \mathrm{~cm}^{-1}$. As can be seen, there is a trend to greater paraffin retention in normal to the cancerous states, metastatic tissue retaining the most paraffin, after the same processing procedures. Further imaging studies are planned to examine the impact of the substrate upon paraffin retention, as there seems to be an interplay of tissue dependence and substrate dependence where the paraffin clumps upon the substrate surface, as can be seen in Figure 5.

Abnormal tissue can be of very different morphology than normal tissue. A difference in tissue density could provide an explanation for the inconsistency of the dewaxing efficiency, and thus the variation of paraffin peak intensity between the normal brain and 
cancerous brain tissue spectra. For breast cancer Li et al. state that the amount of fibroglandular and adipose tissue is strongly related to the risk of developing cancer. They explain that, although no direct evidence has been found to link dense mammographic tissue with the increased chance of developing breast cancer, the correlations observed have encouraged the use of tissue density for breast cancer monitoring. They go on to support the observations of this correlation through their findings that the mammograms of cancerous breast tissue are far denser than those of normal breast tissue ${ }^{37}$. Berrtholdo et al. correlate increased choline signals from Magnetic Resonance Spectroscopy as a measure of malignancy in brain tumours due to increased glial cell density as choline is a marker of cellular membrane turnover reflecting cellular proliferation ${ }^{38}$. Indeed, it should be noted that, whereas in this study complete removal of wax was observed for tissue samples on low-E slides, O' Faoláin et al. ${ }^{39}$ reported incomplete was removal from normal parenchymal tissue from the placenta, dewaxed using a xylene based protocol also measured in low-E slides.

The results indicate that tissue processing still remains an issue for Spectropathology, particularly using Raman spectroscopy. For clinical relevancy, however, and indeed acceptance of the techniques by the clinical community, it is important that the sample preparation and measurement protocols for Raman spectroscopic analyses are consistent with current clinical practice. Choice of substrate for routine clinical analyses may be dominated by economic considerations, thus favouring low E slides. Paraffin embedding facilitates tissue cutting, but also is commonly employed, worldwide, for archiving tissue samples. The availability of a wide range of pathologically characterized samples for study potentially enables extensive retrospective studies using spectroscopic and other techniques. The embedding process and wax itself can, however, contribute significantly to the spectroscopic 
signature of the tissue ${ }^{40}$. An alternate method of "digital dewaxing" biological specimens to remove paraffin contributions of the spectrum has been proposed by Gobinet et al., recognising that chemical dewaxing methods are known to be inefficient for the complete removal of wax in tissue and can cause alterations to the samples. Their method of dewaxing consists of an estimation of the paraffin sources in the spectrum using Independent Component Analysis (ICA), followed by a Non-negatively Constrained Least Squares (NCLS) method to subtract the contributions. This enables removal of the paraffin signals to allow analysis of the underlying tissue spectrum ${ }^{33}$. However, although "digital dewaxing" has been demonstrated ${ }^{41}$, it is also often important to compare spectroscopic profiling with parallel histological analyses, requiring chemical dewaxing of sections, a process which adds to the sample variability. The observation of variable dewaxing efficiencies depending on tissue pathology ads complexity to the challenge, perhaps requiring a combination of established clinical dewaxing protocols with computational procedures.

\section{Conclusion}

This study shows the influence that Raman substrates have on resulting spectra from Raman analysis. Spectral backgrounds of the substrates show that the baseline of low-E is the highest and $\mathrm{CaF}_{2}$ the lowest of the three substrates. It has been established through observation and investigation that $\mathrm{CaF}_{2}$ and Spectrosil substrates retain paraffin wax after the dewaxing procedure is carried out whereas low-E does not. This is attributed to the difference in surface roughness and chemistry between substrates. AFM demonstrated that low-E substrates are far smoother than either $\mathrm{CaF}_{2}$ or Spectrosil substrates with average roughness of $1.10 \mathrm{~nm}$ compared to $4.76 \mathrm{~nm}$ and $3.88 \mathrm{~nm}$ respectively. 
This study has also observed the difference between the dewaxing efficiency between normal brain and cancerous brain tissue. This has been attributed to a change in density between normal and cancerous, which causes the denser cancerous tissue to retain wax better than the, less dense, normal tissue. It is important to note therefore that tissue processing procedures for spectral histopathology of normal tissue may not be optimised for abnormal regions.

\section{Acknowledgements}

The authors would like to acknowledge the support of the Sydney Driscoll Neuroscience Foundation, Rosemere Cancer Foundation, Brain Tumour North West, the Centre for Materials and Science at the University of Central Lancashire, the European Community Action Scheme for the Mobility of University Students and the Association of British Spectroscopists. The Focas Research Institute is supported through the National Biophotonics and Imaging Platform (NBIP) Ireland funded under the Higher Education Authority PRTLI (Programme for Research in Third Level Institutions) Cycle 4, co-funded by the Irish Government and the European Union. The authors thank Dr Alex Henderson for the in-house written Matlab code, Dr Luke O’Neill, Focas Research Institute for assistance with AFM measurements and Mr Reece Hall for assistance with SEM/EDAX measurements.

\section{References}


1. $\quad X$ Prize.

2. S. Frankel, G. D. Smith, J. Donovan and D. Neal, The Lancet, 2003, 361, 1122-1128.

3. R. M. Hoffman and A. Y. Smith, Asian J. Androl., 2011, 13, 369-373.

4. D. Hessels, H. G. Rittenhouse and J. A. Schalken, EAU Update Series, 2005, 3, 200-213.

5. J. B. Lattouf and F. Saad, BJU Int., 2002, 90, 694-699.

6. S. S. Raab, D. M. Grzybicki, J. E. Janosky, R. J. Zarbo, F. A. Meier, C. Jensen and S. J. Geyer, Cancer, 2005, 104, 2205-2213.

7. D. I. Ellis, and R. Goodacre, Analyst, 2006, 131, 875-885.

8. S. E. Taylor, K. T. Cheung, Patel, II, J. Trevisan, H. F. Stringfellow, K. M. Ashton, N. J. Wood, P. J. Keating, P. L. Martin-Hirsch and F. L. Martin, British journal of cancer, 2011, 104, 790-797.

9. R. S. Da Costa, B. C. Wilson and N. E. Marcon, Curr Opin Gastronenterol, 2005, 21, 70-79.

10. M. G. Shim, L.-M. Wong Kee Song, N. E. Marcon and B. C. Wilson, Photochemistry and Photobiology, 2000, 72, 146-150.

11. T. C. Bakker Schut, G. J. Puppels, Y. M. Kraan, J. Greve, L. L. van der Maas and C. G. Figdor, International journal of cancer. Journal international du cancer, 1997, 74, 20-25.

12. J. Sulé-Suso, A. Forster, V. Zholobenko, N. Stone and A. El Haj, Applied spectroscopy, 2004, 58, 61-67.

13. F. M. Lyng, E. Ó. Faoláin, J. Conroy, A. D. Meade, P. Knief, B. Duffy, M. B. Hunter, J. M. Byrne, P. Kelehan and H. J. Byrne, Experimental and Molecular Pathology, 2007, 82, 121-129.

14. A. Podshyvalov, R. K. Sahu, S. Mark, K. Kantarovich, H. Guterman, J. Goldstein, R. Jagannathan, S. Argov and S. Mordechai, Appl. Opt., 2005, 44, 3725-3734.

15. S. Koljenovic, L. P. Choo-Smith, T. C. Bakker Schut, J. M. Kros, H. J. van den Berge and G. Puppels, Lab. Invest, 2002, 82, 1265-1277.

16. C. Beleites, G. Steiner, M. G. Sowa, R. Baumgartner, S. Sobottka, G. Schackert and R. Salzer, Vibrational Spectroscopy, 2005, 38, 143-149.

17. R. Manoharan, K. Shafer, L. Perelman, J. Wu, K. Chen, G. Deinum, M. Fitzmaurice, J. Myles, J. Crowe, R. R. Dasari and M. S. Feld, Photochem Photobiol, 1998, 67, 15-22.

18. R. K. Dukor, M. N. Liebman and B. L. Johnson, Cellular and molecular biology (Noisy-le-Grand, France), 1998, 44, 211-217.

19. M. J. Baker, E. Gazi, M. D. Brown, J. H. Shanks, N. W. Clarke and P. Gardner, Journal of Biophotonics, 2009, 2, 104-113.

20. D. C. Malins, N. L. Polissar and S. J. Gunselman, Proc. Natl. Acad. Sci. USA, 1997, 94, 259-264.

21. M. J. Baker, E. Gazi, M. D. Brown, J. H. Shanks, P. Gardner and N. W. Clarke, British journal of cancer, 2008, 99, 1859-1866.

22. M. Isabelle, N. Stone, H. Barr, M. Vipond, N. Shepherd and K. Rogers, Spectroscopy 2008, 22, 97-104.

23. M. J. Romeo and M. Diem, Vib Spectrosc, 2005, 38, 115-119.

24. C. Kendall, M. Isabelle, F. Bazant-Hegemark, J. Hutchings, L. Orr, J. Babrah, R. Baker and N. Stone, Analyst, 2009, 134, 1029-1045.

25. E. Ó Faoláin, M. B. Hunter, J. M. Byrne, P. Kelehan, H. A. Lambkin, H. J. Byrne and F. M. Lyng, Journal of Histochemistry and Cytochemistry, 2005, 53, 121-129.

26. E. Ó Faoláin, M. Hunter, J. Byrne, P. Kelehan, M. McNamara, H. Byrne and F. Lyng, Materials Synthesis and Applications, Dublin Institute of Technology, 2005, 38, 121-127.

27. F. Bonnier, A. D. Meade, S. Merzha, P. Knief, K. Bhattacharya, F. M. Lyng and H. J. Byrne, Analyst, 2010, 135, 1697-1703.

28. F. Bonnier, P. Knief, A.D. Meade, J. Dorney, K. Bhattacharya, F.M. Lyng, H.J. Byrne, Proc. of SPIE-OSA Biomedical Optics, 2011, SPIE Vol. 8087, 8087F 
29. J. Filik, M. D. Frogley, J. K. Pijanka, K. Wehbe and G. Cinque, Analyst, 2012, 137, 853-861.

30. P. Bassan, J. Lee, A. Sachdeva, J. Pissardini, K. M. Dorling, J. S. Fletcher, A. Henderson and P. Gardner, Analyst, 2013, 138, 144-157.

31. M. Miljkovic, B. Bird, K. Lenau, A. I. Mazur and M. Diem, Analyst, 2013, 138, 3975-3982.

32. J. R. Hands, P. Abel, K. Ashton, T. Dawson, C. Davis, R. Lea, A. Mclntosh and M. Baker, Analytical and Bioanalytical Chemistry, 2013, 1-9.

33. C. Gobinet, D. Sebiskveradze, V. Vrabie, A. Tfayli, O. Piot and M. Manfait,, 16th European Signal Processing Conference (EUSIPCO). 2008.

34. F. Bonnier, S. M. Ali, P. Knief, H. Lambkin, K. Flynn, V. McDonagh, C. Healy, T. C. Lee, F. M. Lyng and H. J. Byrne, Vibrational Spectroscopy, 2012, 61, 124-132.

35. F. Bonnier, A. Mehmood, P. Knief, A. D. Meade, W. Hornebeck, H. Lambkin, K. Flynn, V. McDonagh, C. Healy, T. C. Lee, F. M. Lyng and H. J. Byrne, Journal of Raman Spectroscopy, 2011, 42, 888-896.

36. P. Bassan, A. Sachdeva, J. Lee and P. Gardner, Analyst, 2013, 138, 4139-4146.

37. L. Li, Z. Wu, L. Chen, F. George, Z. Chen, A. Salem, M. Kallergi and C. Berman, Conference proceedings :... Annual International Conference of the IEEE Engineering in Medicine and Biology Society. IEEE Engineering in Medicine and Biology Society. Conference, 2005, 3, 32533256.

38. D. Bertholdo, A. Watcharakorn, M. Castillo, Neuroimaging Clinics, 2013, 23(3), 359-380

39. E.O. Faolain, M.B. Huinter, J.M. Byrne, P. Kelehan. M. Mcnamara, H.J. Byrne, F.M. Lyng, Vibrational Spectroscopy ${ }_{\llcorner} 2005,38$ (1-2), 121-127

40. E.O. Faolain, M.B. Huinter, J.M. Byrne, P. Kelehan. P. Lambkin, H.J. Byrne, F.M. Lyng Faolain, J Histochem Cytochem, 2005, 53, 121-129

41. A. Tfayli, C. Gobinet, V. Vrabie,R. Huez, M. Manfait,O. Piot, App/ Spectrosc, 2009, 63, 564570

\section{List of Tables and Figures}

Table 1 Patient details with histological subtype and metastatic origin.

Figure 1 Microscopic images of H\&E stained tissue samples of normal brain, metastatic (from oesophagus and stomach, breast, colon/rectum, lung and melanoma as labelled above) and GBM brain WHO grade IV (x 100). Scale bar represents 50 microns.

Figure 3 Averaged spectra of $458 \mathrm{CaF}_{2}$ acquisitions, 210 low-E acquisitions and 465 Spectrosil acquisitions from all tissue specimens and a background-subtracted spectrum of paraffin wax. The tissue spectra have been vector normalised and offset for visual clarity. The asterisks above the peaks correspond to the paraffin contributions.

Figure 4 Vector normalised, background corrected spectra of substrates after dewaxing from: $1 \mathrm{CaF}_{2}$ acquisition, 1 Spectrosil acquisition and 10 averaged low-E acquisitions. The spectrum of paraffin wax is included as a reference. The spectra have been appropriately scaled and offset for visual clarity.

Figure 5 Microscopic Raman images at x 10 of the substrate surfaces after dewaxing. Wax residuals can be observed on the $\mathrm{CaF}_{2}$ and Spectrosil substrates. Scale bars represent $0.5 \mathrm{~mm}$. 
Figure 6 AFM images of Spectrosil, $\mathrm{CaF}_{2}$ and Low-E substrates.

Figure 7 Averaged immersion spectra of 127 GBM acquisitions, 668 metastatic acquisitions and 157 normal brain acquisitions. Normal tissue spectrum is shown with its standard deviation in red (top), GBM tissue spectrum is shown with its standard deviation in green (middle), and metastatic tissue spectrum is shown with its standard deviation in blue (bottom). Spectra have been vector normalised, the backgrounds have been corrected using a 5 th order polynomial fit and subtraction and 7 points of smoothing. The asterisks correspond to paraffin peaks from residual wax in the tissue.

Figure 8 The average intensity and standard deviation of three intense paraffin peaks at 1061, 1131 and $1294 \mathrm{~cm}^{-1}$ for the three tissue types; Normal tissue (blue), Glioblastoma multiforme (red) and Metastatic brain cancer (green) 